\title{
MALARIA BURDEN IN HUMAN POPULATION OF QUETTA, PAKISTAN
}

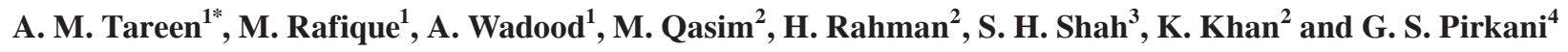 \\ ${ }^{1}$ Department of Microbiology, University of Balochistan, Quetta, Pakistan \\ ${ }^{2}$ Department of Microbiology, Kohat University of Science and Technology, Kohat, Pakistan \\ ${ }^{3}$ Department of Statistic, University of Balochistan, Quetta, Pakistan \\ ${ }^{4}$ Department of Microbiology, Bolan Medical College, Quetta, Pakistan
}

Received: April 11, 2012; Accepted: May 21, 2012

\begin{abstract}
Malaria is a serious global health challenge, which is responsible for more than one million deaths a year. Malarial infection is more prevalent in developing countries including Pakistan. Significant efforts have been made to control malaria; however, due to socioenvironmental factors, it remains a frequent problem in Quetta. The present study was undertaken to determine the malarial incidence, species prevalence, and its demographic evaluation in human population of Quetta, Pakistan. A total of 1831 subjects, comprising 1072 male and 759 female presenting symptoms of malaria, were included in this study. Blood samples from clinically suspected individuals were subjected to the standard immunochromatographic and malaria parasite smear analysis for malaria diagnosis. Out of 1831 subjects, $338(18.45 \%)$ patients were positive for malarial parasite while the species prevalence was found as $276(81.66 \%)$ and $62(18.34 \%)$ for Plasmodium vivax, and Plasmodium falciparum, respectively. Furthermore, seasonal variations gradual increase in the prevalence rate. The age group of 21-30 years (30.47\%) was found more prone to malaria. The suspected malaria cases were found more frequent in rural (72.1\%) as compared to urban (27.9\%). In addition, the malaria burden was high in urban area (22.89\%) population as compared to the rural area $(16.74 \%)$ population. It was observed that the highest disease occurrence was caused by $P$. vivax, which reflects a serious threat for public health. The current findings will be helpful to plan effective strategies to prevent and control malaria in this area.
\end{abstract}

Keywords: malaria, Quetta, prevalence rate

\section{Introduction}

Malaria is a serious public health problem which represents the fifth leading cause of death from infectious disease worldwide [1], which accounts for about one million deaths every year [2]. The malarial control economical burden is alarming as the annual cost for financial assistance to malaria-endemic countries has been rising from approximately $\$ 100$ million annually to nearly $\$ 1.8$ billion during 2003-2010 [1]. In addition, a global estimated budget of US\$ 5 billion a year between 2010 and 2015 and US\$ 4.75 billion between 2020 and 2025 will be required to control malaria [3]. Malarial problem mainly persists in tropical and subtropical regions, as their temperature and rainfall favor the survival of malaria-causing Plasmodium parasites [4]. It has been found that $7 \%$ of the $\mathrm{Pa}-$ kistani population is at risk of contracting malaria, with an estimated nationwide burden of 1.6 million cases per year [5]. In 2009, 4.2 million malaria cases were reported in Pakistan. Among all confirmed cases, Plasmodium falciparum accounts for about $30 \%$ while $42 \%$ of the confirmed cases are originated in Balochistan province [5]. Recent reports revealed that Pakistan is endemics for both Plasmodium vivax and P. falciparum $[6,7]$ accounting for $75 \%$ and $25 \%$ malarial cases, respectively [8]. The predisposition factors include uncontrolled migration of Afghan refugees, urbanization, an expansive irrigation network, uncontrolled population growth rate, sudden environmental changes due to excessive and monsoon rains, earth quakes, floods, and water development projects making the environment of Pakistan favorable for persistence of malarial species [8]. In addition, malnutrition, low individual immunity, or passage to high transmission areas, overstretched health infrastructure, and unhygienic camps providing mosquito breeding places are contributing factors for increased incidence of malaria transmission in Pakistan especially in Afghan refugee camps [6, 9]. Malaria is associated with seasonal variations as most of the malarial infection occurs in September and November, following the monsoon season [8]. Furthermore, epidemic outbreaks in particular geographical areas, especially in the provinces of Balochistan, Sindh, Khyber Pakhtunkhwa, and the federally administered tribal areas have the highest malaria burden [8]. Due to malarial control programs operated by several organizations in the world, the estimated number of malarial cases reduced

\footnotetext{
*Corresponding author: Dr. Abdul Malik Tareen; Department of Microbiology, University of Balochistan, Quetta, Pakistan; Phone: +92-81-9211004; E-mail: abdulmaliktareen@yahoo.com
} 
from 244 million in 2005 to 225 million in 2009 and associated with a $21 \%$ decrease in estimated global malaria deaths from 2000 to 2009 [1, 5].

In the past, the malaria research was confined to $P$. falciparum infection as compared to $P$. vivax due to its high mortality rate in various parts of the world. Nowadays, $P$. vivax has been accepted as an emerging threat to human, as it is considered a major cause of malaria morbidity outside Africa, especially Asia and the Americas. In addition, $P$. vivax is an alarming threat to almost $40 \%$ of the world's population, including 132 to 391 million clinical infections annually $[10,11]$.

The present study was carried out in Quetta district of Balochistan, Pakistan an endemic malaria region to identify the prevalence of malarial vectors and to identify major risk factors associated with parasitemia along with socioeconomic and geographical patterns risk factors. The undertaken study might be helpful to plan cost-effective interventions for malaria control.

\section{Materials and methods}

\section{Study population}

The prospective study was conducted on 1831 clinically suspects in a span of 4 months from January 2011 to April 2011 in Bolan medical complex hospital (BMC), Quetta, Pakistan. Quetta is on the Western edge of Pakistan and provincial capital of Balochistan. It is located at $30^{\circ} 12^{\prime}$ $38^{\prime \prime} \mathrm{N}, 67^{\circ} 1^{\prime} 8^{\prime \prime} \mathrm{E}$ at an average altitude of $1654 \mathrm{~m}$ above sea level. Quetta, which sits near Durand line border with Afghanistan and Taftan with Iran, is an important trade and communication center. Average population of the city according to 2001 censuses is 896,090 . Average temperature of city is approximately $38^{\circ} \mathrm{C}$, and average rain fall is $32 \mathrm{~mm}$, lacking moon soon season of sustained heavy rain fall, supporting the breeding of mosquitoes.

The following inclusion criteria were used to select samples in this study: age ( 1 to 70 years) from both sexes and clinical features of shaking chills, high fever, sweating associated with fatigue, headache, dizziness, nausea, vomiting, abdominal cramps, dry cough, muscle pain, and back ache.

\section{Sample collection}

The detailed history (age, gender, area, and clinical signs) of all suspected individuals was documented. The blood samples of malarial suspects were collected aseptically according to standard protocols and with patient consent. Blood samples were screened for malarial parasite through rapid routine serological test and microscopic examination of thick and thin blood smears slides in Bolan medical complex hospital (BMC), Quetta, Pakistan. All samples were processed without delay.

\section{Malaria diagnosis}

\section{Rapid diagnostic test}

The malaria pflpan rapid test was performed according to the manufacturer's instructions. This is a rapid chromatographic immunoassay [12] for the detecting P. falciparum, $P$. vivax, Plasmodium ovale, and Plasmodium malariae antigen in the whole blood.

\section{Microscopic analysis}

Slide preparation technique was also preformed to detect the type and stage of the malarial parasite. Thick and thin blood films were prepared on clean glass slides, fixed, and Giemsa stained [13]. Thin films were used for to categorize the patients as mild, moderate, high, and very high parasite density. In addition, thick smear was examined under oil immersion lens of the microscope, and malarial parasites species were counted against 300 white blood cells (WBCs). On the basis of microscopic features, malaria species were identified.

\section{Results and discussion}

The obtained data from suspected cases were checked according to the season, age, gender, and area in human population of Quetta district. Among the total suspected malaria cases (1831), a total of $81.50 \%$ suspected cases (1493) were found to be negative for malaria parasite. The overall incidence of malaria was found to be $18.45 \%$ (338) (Fig. 1). In our data, the male was more affected than female. The incidence of $P$. vivax was more in male (52.66\%) (Fig. 2).

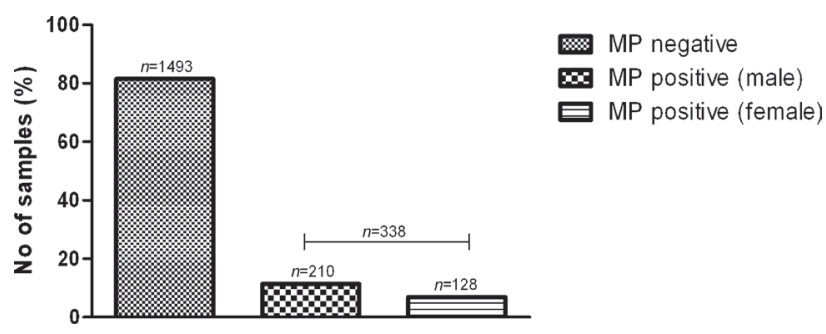

Fig. 1. Incidence of malaria among the suspected individuals

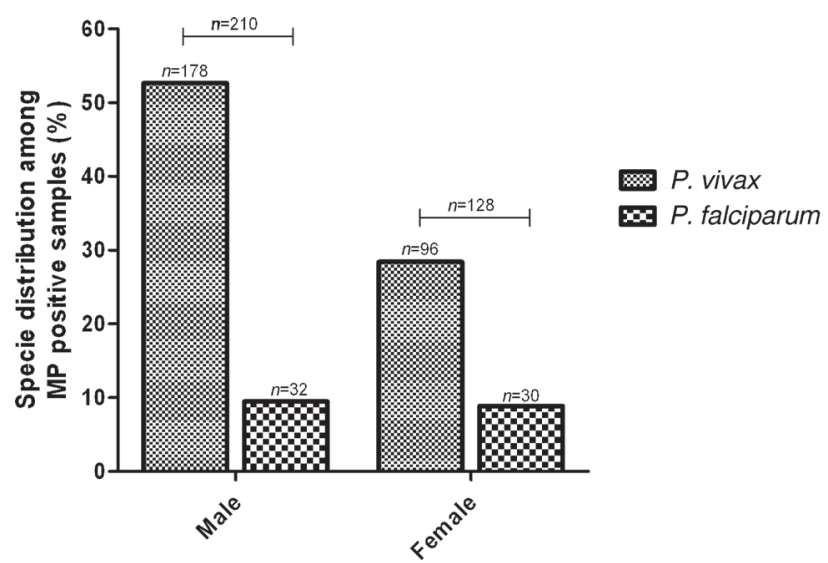

Fig. 2. Prevalence of malaria species with respect to gender 


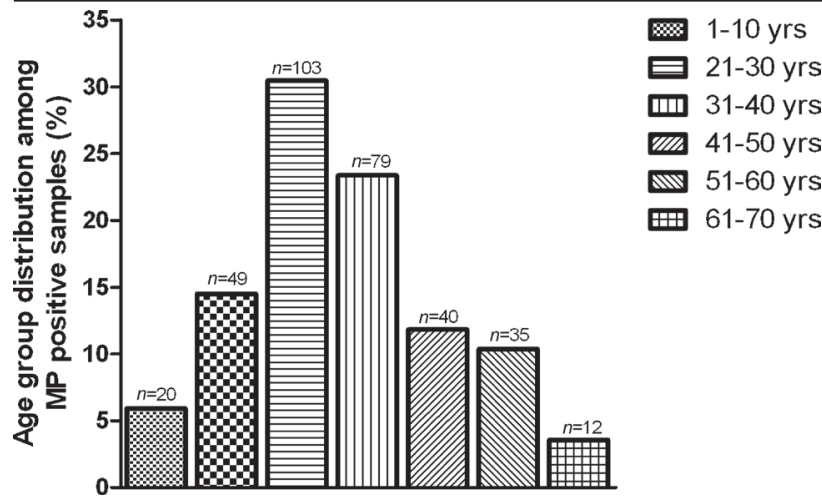

Fig. 3. Malaria incidence in various age group of human population in Quetta district

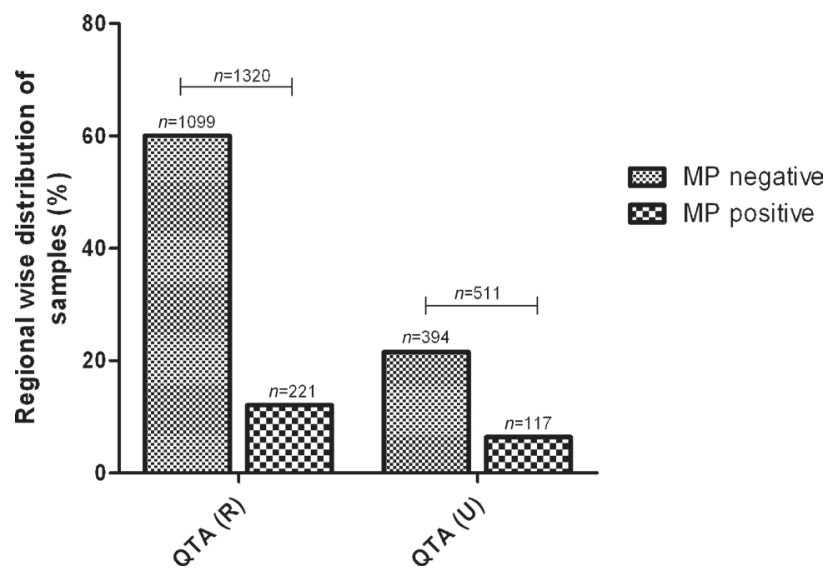

Fig. 4. Malaria incidence in rural and urban area

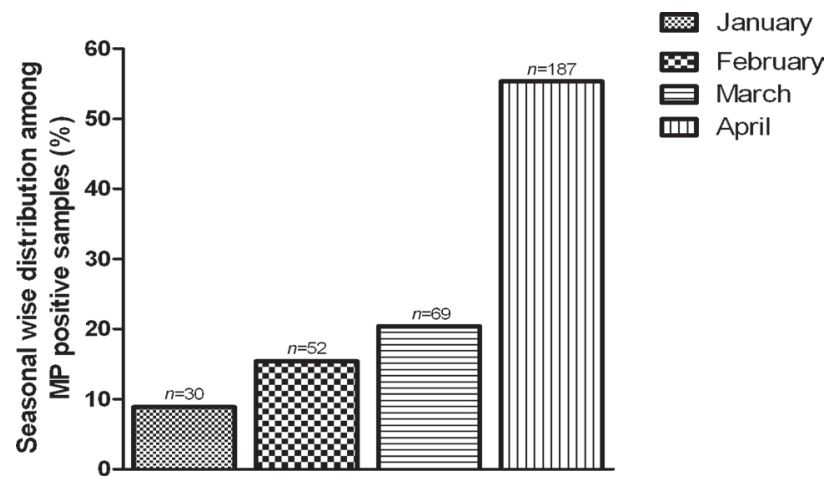

Fig. 5. Seasonal variations affect on malaria distribution

In order to find the involvement of age factor on malaria incidence, the data showed high rate of malaria affecting the adults of age group 21-30 years (30.47\%) (Fig. 3).

To figure out any difference of disease burden in the urban and rural area of Quetta, the study was divided into 2 groups and data were collected from respective areas. The incidence of malaria positive cases was found more frequent in urban $(22.89 \%, n=117)$ as compared to rural $(16.74 \%, n=221)$ (Fig. 4). Seasonal variation pattern was also documented which exhibits a gradual increase in the malaria incidence from January (8.88\%) to April (55.33\%) (Fig. 5).

In global perspective, the rate of mortality and morbidity associated with malaria is a major challenge to public health. According to the recent WHO report, about half of the world's population is at risk of malaria, and an estimated 250 million cases led to nearly 1 million deaths in 2006 [5]. Among them, Pakistan is also endemic for both $P$. vivax, and $P$. falciparum associated malaria infections $[6,14,15]$.

The present study was conducted in Quetta district which has the highest rate of malaria prevalence $[6,16]$. Among all the suspected patients $(n=1831)$, the rate of malaria burden we found was $18.45 \%(n=338)$, which is in line with the previous study executed in other parts of Balochistan [6, 16].

According to this data, male $(11.46 \%, n=210)$ was more prone to malaria than female $(6.99 \%, n=128)$ (Fig. 1). The predominance malaria prevalence in males in our study can be justified by the fact that males have more exposures to malaria vector than females due to their activities, as they work in mines or fields at peak biting times or migrate to areas of high endemicity for work. Another explanation might be restricted mobility of female in Quetta district. A similar investigation in Pakistan reported malaria incidence higher in male in contrast to female patients [17]. Our findings are also consistent with another study in Iran which showed high prevalence of malaria in male as compared to female [18].

In addition, $P$. vivax was the main causative agent of malaria in male $(52.66 \%, n=178)$ and in female $(28.42 \%$, $n=96$ ) population as compared to P. falciparum (Fig. 2). Previous studies conducting in other parts of Pakistan reported higher prevalence rate of $P$. vivax infection as compared to $P$. falciparum $[6,19,20]$. $P$. vivax is more commonly distributed in temperate zones and is more prevalent than $P$. falciparum [21]. It is believed that $P$. vivax has the greater ability to produce gametocytemia under temperate conditions [22]. Furthermore, the possible explanation will be the climate change which might be responsible for the natural distribution of malaria [23, 24] associated with $P$. vivax in this area. Though some groups reported the mixed infections of $P$. vivax and $P$. falciparum $[20$, $25,26]$, however, we have not observed any case of mixed infection in our study.

In order to document the age factor on the malaria incidence, it was found that malaria can affect all the age groups; however, the age group between 21 and 30 years was more susceptible to malaria infection (30.47\%) (Fig. 3 ). These findings are in line with the previous study which reported $66.1 \%$ of malaria cases in the age group above 14 years [18]. The people belonging to this age group are involving in different professional activities and have greater exposure to mosquitoes bite as they remained outside during most part of day.

The clinically suspected malaria cases in the rural area of Quetta were at highest rate $(n=1320)$ as compared to urban region $(n=511)$ while, interestingly, the percent positivity was higher in urban $(22.89 \%, n=117)$ when compared to rural $(16.74 \%, n=221)$ region (Fig. 4). Moreover, we found gradual increase in malaria incidence. Malaria was more prevalent in April (55.33\%) while least prevalence was documented in the month of January (8.88\%) 
(Fig. 5). It is already reported that the gradual increase in temperature was favoring the development and multiplication of Plasmodium species [21]; thus, temperature increase in the month of April might be one reason of malaria spread in the Quetta district.

In conclusion, our study reported the highest disease burden caused by $P$. vivax, which reflects a serious threat for public health. The findings of the undertaken study will be helpful to setup an emergent anti-malarial campaign to prevent and control the spread of malaria in this area.

\section{Acknowledgements}

We thank to Mr. Ghaffar Zari and Lab Technician Nisar Kurd for providing technical assistance.

\section{References}

1. Centers for Disease Control and Prevention (CDC): Ten great public health achievements - worldwide, 2001-2010. MMWR Morb Mortal Wkly Rep 60, 814-818 (2011)

2. Greenwood BM, Bojang K, Whitty CJ, Targett GA: Malaria. The Lancet 365, 1487-1498 (1923)

3. World Health Organization Geneva: The global malaria action plan (Roll Back Malaria) 2008. http://wwwrollbackmalaria org/gmap 2008.

4. Greenwood BM, Fidock DA, Kyle DE, Kappe SHI, Alonso PL, Collins FH: Malaria: progress, perils, and prospects for eradication. The Journal of Clinical Investigation 118, 12661276 (2008)

5. World Health Organization: World Malaria Report 2010. http://www who int/malaria/world_malaria_report_2010/ worldmalariareport2010 pdf 2010.

6. Yasinzai MI, Kakersulmankhal KJ: Incidence of human malaria infection in northern hilly region of Balochistan, adjoining with NWFP, Pakistan: district Zhob. Pak J Biol Sci 11, 1620-1624 (2008)

7. Asif SA: Departmental audit of malaria control programme 2001-2005 North West Frontier Province (NWFP). J Ayub Med Coll Abbottabad 20, 98-102 (2008)

8. Khatoon L, Baliraine F, Bonizzoni M, Malik S, Yan G: Genetic structure of Plasmodium vivax and Plasmodium falciparum in the Bannu district of Pakistan. Malaria Journal 9, $112(2010)$

9. UK Department for International Development: Malaria: Burden and Interventions 2010. http://www dfid gov uk/Documents/prd/malaria-evidence-paper pdf 2010.
10. Price RN, Tjitra E, Guerra CA, Yeung S, White NJ, Anstey NM: Vivax malaria: neglected and not benign. Am J Trop Med Hyg 77, 79-87 (2007)

11. Carlton JM, Adams JH, Silva JC, Bidwell SL, Lorenzi H, Caler E. et al.: Comparative genomics of the neglected human malaria parasite Plasmodium vivax. Nature 455, 757-763 (2008)

12. Harani MS, Beg MA, Khaleeq L, Adil SN, Kakepoto GN, Khurshid M: Role of ICT malaria immunochromatographic test for rapid diagnosis of malaria. J Pak Med Assoc 56, 167171 (2006)

13. Jager MM, Murk JL, Pique RD, Hekker TA, VandenbrouckeGrauls CM: Five-minute Giemsa stain for rapid detection of malaria parasites in blood smears. Trop Doct 41, 33-35 (2011)

14. Suleman M: Malaria in Afghan refugees in Pakistan. Trans $R$ Soc Trop Med Hyg 82, 44-47 (1988)

15. Zakeri S, Kakar Q, Ghasemi F, Raeisi A, Butt W, Safi N et al.: Detection of mixed Plasmodium falciparum \& P. vivax infections by nested-PCR in Pakistan, Iran \& Afghanistan. Indian J Med Res 132, 31-35 (2010)

16. Yasinzai MI, Kakarsulemankhel JK: Prevalence of human malaria infection in bordering areas of East Balochistan, adjoining with Punjab: Loralai and Musakhel. J Pak Med Assoc 59, 132-135 (2009)

17. Khan HU, Khattak AM, Khan MH, Mahsud IU, S. Humayun S: A study of prevalence of malaria in adult population of D. I. Khan, Pakistan. Biomedica 22, 99-104 (2006)

18. Youssifi MR, Rahimi MT: Prevalence of malaria infection in Sarbaz, Sistan and Baluchistan province. Asian Pacific Journal of Tropical Biomedicine, 491-492 (2011)

19. Jalal UD, Khan SA, Ally SH: Malaria in children: study of 160 cases at a private clinic in Mansehra. J Ayub Med Coll Abbottabad 18, 44-45 (2006)

20. Idris M, Sarwar J, Fareed J: Pattern of malarial infection diagnosed at Ayub Teaching Hospital Abbottabad. J Ayub Med Coll Abbottabad 19, 35-36 (2007)

21. Patz JA, Olson SH: Malaria risk and temperature: influences from global climate change and local land use practices. Proc Natl Acad Sci U S A 103, 5635-5636 (2006)

22. Strickland GT, Zafar-Latif A, Fox E, Khaliq AA, Chowdhry MA: Endemic malaria in four villages of the Pakistani province of Punjab. Trans R Soc Trop Med Hyg 81, 36-41 (1987)

23. Tonnang HE, Kangalawe RY, Yanda PZ: Predicting and mapping malaria under climate change scenarios: the potential redistribution of malaria vectors in Africa. Malar J 9, 111 (2010)

24. Reiter P: The standardised freight container: vector of vectors and vector-borne diseases. Rev Sci Tech 29, 57-64 (2010)

25. McKenzie FE, Bossert WH: Multispecies Plasmodium infections of humans. J Parasitol 85, 12-18 (1999)

26. McKenzie FE, Bossert WH: Mixed-species Plasmodium infections of humans. J Parasitol 83, 593-600 (1997) 